\title{
Aprendizaje Temprano a la Ingesta de Concentrado en Alpacas Huacaya
}

\author{
Early Learning to Concentrate Intake in Huacaya Alpacas
}

Jorge Castro Bedriñana ${ }^{1,3}$, Doris Chirinos Peinado ${ }^{1}$, Robert Rojas Pérez ${ }^{1,2}$

\section{Resumen}

El estudio tuvo como objetivo determinar la edad de alpacas Huacaya en el aprendizaje temprano a la ingesta de concentrado con influencia materna previa, sustentado en la susceptibilidad de las alpacas jóvenes de no ingerir concentrados a causa de la neofobia. El estudio se llevó a cabo en la sierra central del Perú, con 30 crías de alpaca (y sus madres), distribuidas en tres grupos de 10 animales de 2, 3 y 4 meses de edad por cada grupo. Se les ofreció un concentrado por 15 días a base de subproducto de trigo, cebada molida, pasta de algodón, melaza y sales minerales, y se evaluó la aceptación diaria. El $80 \%$ de las crías de cuatro meses aceptaron el concentrado a los $8.8 \pm 0.5$ días $(\mathrm{p}<0.05)$. Al día 11, 4 de las 10 crías de dos meses de edad aceptaron el concentrado, y al día 13 todas aceptaron el concentrado, mientras que todas las crías de tres meses de edad aceptaron el concentrado al día 11. La uniformización del consumo de concentrado fue mejor en las crías de cuatro meses con $10.0 \pm 1.0$ días, siendo para los de 2 y 3 meses a los $12.6 \pm 0.9$ y $12.0 \pm 1.0$ días, respectivamente $(\mathrm{p}<0.05)$. El consumo voluntario fue mayor en las crías de tres meses con $56.7 \pm 18.5 \mathrm{~g}(\mathrm{p}<0.05)$, evidenciando que la mejor edad para el aprendizaje temprano de las alpacas al consumo de concentrado fue a los tres meses de edad.

Palabras clave: neofobia, influencia materna, alpacas, aceptación al concentrado, camélidos sudamericanos

\section{Abstract}

The study aimed to determine the age of Huacaya alpacas in early learning to concentrate intake under maternal influence, based on the susceptibility of young alpacas of not eating concentrates due to neophobia. The study was conducted in the central highlands of Peru, with 30 baby alpaca (and their mothers), divided into three groups of

\footnotetext{
${ }^{1}$ Facultad de Zootecnia, Universidad Nacional del Centro del Perú, Huancayo, Perú

${ }^{2}$ In memoria (QEPD)

${ }^{3}$ E-mail: jorgecastrobe@yahoo.com
}

Recibido: 10 de junio de 2016

Aceptado para publicación: 3 de noviembre de 2016 
10 animals of 2, 3 and 4 months of age per group. The baby alpacas were offered a concentrate for 15 days based on wheat byproduct, ground barley, cotton pulp, molasses and minerals and the daily acceptance was assessed. Eighty per cent of 4-month animals accepted the concentrate at $8.8 \pm 0.5$ days $(\mathrm{p}<0.05)$. At day 11 , only 4 of the 2 -month animals accepted the concentrate, but all accepted it at day 13, while all 3-month old animals accepted concentrate at day 11. Concentrate intake was standardized at an earlier stage (10.0 \pm 1.0 days) in 4-month old while for the 2 and 3-month old animals were at 12.6 \pm 0.9 and $12.0 \pm 1.0$ days respectively $(\mathrm{p}<0.05)$. Voluntary intake was higher in 3 -month old animals $(56.7 \pm 18.5 \mathrm{~g} ; \mathrm{p}<0.05)$, showing that the best age for early learning concentrate consumption was three months.

Key words: neophobia, maternal influence, alpacas, concentrate acceptance, South American camelids

\section{INTRODUCCIÓN}

La fisiología digestiva de las alpacas se caracteriza por una alta susceptibilidad al cambio de manejo y composición de la dieta a la cual no están familiarizadas. Este fenómeno es denominado neofobia y se manifiesta como un comportamiento cauteloso con relación a nuevos alimentos, lo cual podría ser superado por el acompañamiento materno y la presencia de alimentos familiares al animal (Provenza, 2003b).

En los herbívoros jóvenes, prestar atención a la madre es decisivo para aprender qué comer, teniendo gran habilidad para recordar por varios años los alimentos que deben consumir (Launchbaugh y Provenza, 1991; Provenza, 2003b). Estas experiencias tempranas provocan cambios neurológicos que explicarían por qué algunos hábitos son difíciles de romper (Provenza et al., 1992; Provenza y Launchbaugh, 1999), siendo los animales jóvenes más influenciados por la madre (Mirza y Provenza, 1994).

El acondicionamiento de preferencias por los alimentos, según los efectos que los alimentos causan dentro del animal, según la memoria moldeada por el medio ambiente, puede ser de diferentes tipos: (1) familiar aversivo: alimentos ingeridos anteriormente relacionados con un malestar producido después de su ingestión; (2) nuevo: alimentos que nunca han sido consumidos por el animal; (3) familiar sano: alimentos que no causan problema alguno después de su ingesta; y (4) familiar posesivo: alimentos relacionados con la recuperación de algún mal (Provenza, 1995, 2003a).

Provenza y Villalba (2006) indican que los animales al nacimiento tienen que aprender qué comer y qué comida evitar. Provenza (2003b) señala que todos los animales son neofóbicos ante la presencia de alimentos nuevos, de allí que se debe iniciarles comiendo cantidades pequeñas de alimentos nuevos, de modo que irán incrementando su consumo si son nutritivos (Provenza, 1995), especialmente si comen junto a sus madres (Provenza, 2003a,b).

Las alpacas, ante cambios en el tipo o presentación de alimentos con los que no están familiarizadas, reducen abruptamente su consumo por un tiempo más prolongado que el ovino y la llama, antes de recuperar su nivel normal de consumo (Provenza y Balph, 1987). Es así que el entrenamiento predestete es una buena estrategia para reducir la neofobia y promocionar la familiarización y aceptación de los alimentos nuevos (Dam Van Tien y Ha, 2000; Dam Van Tien, 2003). 
Si bien se reconoce la dependencia alimentaria de los camélidos sudamericanos de las praderas altoandinas, los esfuerzos realizados para entender su fisiología digestiva permitirán diseñar algunas alternativas alimentarias que mejoren su rendimiento productivo (San Martín, 1994). En este sentido, con la finalidad de una mejor comprensión del comportamiento de las crías de alpacas con respecto a la aceptación de alimentos nuevos, el presente estudio tuvo como objetivos determinar, en la alpaca, la edad en que se registra el menor tiempo de aceptación de concentrado, la edad en que uniformizan en menor tiempo el consumo de concentrado y la edad en que consumen la mayor cantidad de concentrado por día.

\section{Materiales y MéTodos}

El presente estudio se llevó a cabo entre enero y junio de 2008 en la Unidad de Producción de Conocancha de la Sociedad Agraria de Interés Social Pachacútec, ubicada en el distrito de Marcopomacocha, departamento de Junín, Perú, a 4200 msnm. La fase de campo tuvo un periodo de seis meses.

La unidad de producción tenía cerca de 15000 alpacas Huacaya, habiéndose utilizado una muestra de 30 alpacas machos de 2 , 3 y 4 meses de edad (10 animales por edad), con sus respectivas madres, los cuales fueron alojados en corrales individuales de $3 \times 3$ $\mathrm{m}$. El trabajo se inició desde el momento del nacimiento de los animales, distribuyendo en forma randomizada a las crías macho en tres grupos para evaluarlas cuando cumplan las edades antes señaladas. Para este fin, fueron identificadas con aretes de diferente color.

El estudio tuvo cuatro etapas. La primera consideró un periodo de 15 días donde las madres recibieron el concentrado de estudio luego del parto y por tres horas diarias previas al pastoreo (08:00-11:00), a fin de que las crías se familiaricen con el concentrado por medio del olor y el sabor transmitido en la leche materna. Las siguientes etapas se realizaron cuando las crías cumplieron los 2,3 y 4 meses de edad. El agua fue administrada a discreción.

Las crías, una vez cumplidas las edades requeridas (2, 3 o 4 meses de edad), tuvieron acceso durante 15 días y sin la presencia de la madre, al mismo concentrado que sus madres consumieron al inicio de su lactancia. El concentrado estuvo disponible por tres horas diarias (08:00-11:00), para luego pasar al pastoreo junto con sus madres.

Los comederos fueron de material plástico y de color uniforme. La cantidad diaria de concentrado ofrecido estuvo acorde al peso promedio de cada grupo de crías. Los pesos promedio de los animales de 2, 3 y 4 cuatro meses de edad fueron de $10.7 \pm 0.9$, $13.1 \pm 0.9$ y $15.5 \pm 0.9 \mathrm{~kg}$, calculando el consumo diario de alimento en base al 1.8\% del peso vivo (San Martín, 1994), siendo de 200 , 250 y $300 \mathrm{~g} /$ cría/día, respectivamente. Los animales fueron pesados utilizando una balanza colgante tipo reloj, de $25 \mathrm{~kg}$ de capacidad con escala de $100 \mathrm{~g}$.

Durante los 15 días en que las crías tuvieron acceso al concentrado, se evaluó (a) el tiempo de aceptación del concentrado, registrando su consumo hasta el momento de consumo voluntario; es decir, el momento en que los animales mantienen un consumo uniforme de concentrado; (b) la uniformidad en el consumo dado por el tiempo en que los animales mantuvieron un consumo uniforme sin disminución significativa del consumo, $\mathrm{y}$ (c) el consumo neto de concentrado, que fue determinado por diferencia entre la cantidad ofrecida y el residuo.

El alimento fue pesado a las 08:00, hora en que se colocó en los comederos, y el residuo se pesó a las 11:00, hora en que los animales salieron al pastoreo. Para el pesaje del concentrado se utilizó una balanza electrónica de $5000 \pm 2 \mathrm{~g}$. 
El concentrado fue elaborado con subproducto de trigo, cebada molida y torta de soya molida y contenía $13.62 \%$ de proteína digestible, $5.4 \%$ de fibra cruda y $61.2 \mathrm{Kcal}$ de energía digestible por $\mathrm{kg} \mathrm{W}^{0.75}$, calculado según los reportes de San Martín (1991) y López y Raggi (1992). El consumo de nutrientes según el peso metabólico consideró $9.33,10.57$ y $11.77 \mathrm{~g}$ de proteína digestible diaria para los animales de 2, 3 y 4 meses de edad, cuyos pesos metabólicos fueron de $6.88,7.79$ y $8.68 \mathrm{~kg}$. Para tener un aporte adecuado de minerales, se incorporó una premezcla comercial de vitaminas y minerales, que incluía, además, melaza y bentonita. La bentonita actúa como atrapante de toxinas y la melaza en polvo como energía de rápido uso, previniendo el estrés y la incidencia de alguna alteración en el estado de sistema digestivo, así como evitar la formación de la aversión al alimento ofrecido (Burritt y Provenza, 1991, 2000; Provenza et al., 1992).

En el estudio se utilizó un diseño completamente randomizado con tres tratamientos (edades) y 10 repeticiones (crías) por tratamiento, determinándose las diferencias estadísticas entre promedios mediante pruebas de significación de Tukey, con el software SPSS 17.

\section{Resultados y Discusión}

El tiempo de aceptación al concentrado varió de acuerdo a la edad (Cuadro 1). Las crías de 2 y 3 meses de edad presentaron una mayor variabilidad individual, siendo entre 6 y 11 y entre 8 y 13 días, respectivamente. Solo el $80 \%$ de las crías de cuatro meses aceptaron el alimento casi uniformemente (entre el día 8 y 9), pero el 20\% no lo llegó a aceptar dentro del periodo de 15 días del estudio, indicando que la neofobia comenzaba a mostrarse. Estos resultados están en relación con varios autores (Provenza, 1995; Provenza y Launchbaugh, 1999; Dam Van Tien y Ha, 2000; Dam Van Tien, 2003;
Cuadro 1. Tiempo de aceptación del concentrado según la edad de las crías de alpaca ( $\mathrm{n}=10$ por grupo)

\begin{tabular}{cc}
\hline $\begin{array}{c}\text { Grupos } \\
\text { (meses de edad) }\end{array}$ & $\begin{array}{c}\text { Aceptación del } \\
\text { concentrado (días) }\end{array}$ \\
\hline 2 & $11.2 \pm 1.7^{\mathrm{a}}$ \\
3 & $9.6 \pm 2.1^{\mathrm{ab}}$ \\
4 & $8.8 \pm 0.5^{\mathrm{b}}$ \\
\hline $\mathrm{a}, \mathrm{b}, \mathrm{c}$ Superíndices diferentes indican diferencia \\
estadística $(\mathrm{p}<0.05)$
\end{tabular}

Provenza, 2003a), quienes dicen que el mejor resultado al consumo está dado por el aprendizaje temprano, pues el aprendizaje a través de las señales en la leche de la madre establece una preferencia de alimentos, ayudando a los animales a adaptarse a su entorno alimentario después del destete.

En el Cuadro 2 se muestra la distribución del consumo promedio y el número de animales por tratamiento que aceptan el alimento en el día 11 del estudio. El consumo promedio en las crías de tres meses de edad fue significativamente superior en comparación con los otros grupos $(\mathrm{p}<0.01)$.

Los promedios de días en que las unidades experimentales uniformizan el consumo de concentrado se muestran en el Cuadro 3. Los resultados indican que en los animales de mayor edad que consumieron el concentrado, uniformizaron el consumo antes que los demás.

El consumo promedio diario de concentrado durante los 15 días de la fase de exposición fue mayor en los animales de tres meses de edad con respecto a los animales de cuatro meses de edad $(\mathrm{p}<0.05)$, aunque con una mayor variabilidad (Cuadro 4). El consumo promedio de los animales de 2 y 4 meses representó el 81.6 y $60.7 \%$ del consumo re- 
Cuadro 2. Distribución de consumo promedio de concentrado (g) y número de alpacas, según edad, que aceptan el concentrado en el día 11 de la suplementación

\begin{tabular}{lccc}
\hline & $\begin{array}{c}2 \text { meses } \\
(\mathrm{n}=10)\end{array}$ & $\begin{array}{c}3 \text { meses } \\
(\mathrm{n}=10)\end{array}$ & $\begin{array}{c}4 \text { meses } \\
(\mathrm{n}=10)\end{array}$ \\
\hline $\begin{array}{l}\text { Crías que aceptan }(\mathrm{n}) \\
\text { Consumo de alimento (g/día) }\end{array}$ & 4 & 10 & 8 \\
\hline${ }^{\mathrm{a}, \mathrm{b}}$ Superíndices diferentes indican diferencia estadística $(\mathrm{p}<0.05)$ & & \\
$\begin{array}{l}\text { Chi-cuadrado }=9.93 \\
\end{array}$
\end{tabular}

Cuadro 3. Tiempo en que se uniformiza el consumo de concentrado, según la edad de las crías de alpaca $(\mathrm{n}=10$ por grupo)

\begin{tabular}{cc}
\hline $\begin{array}{c}\text { Grupos } \\
\text { (meses de edad) }\end{array}$ & $\begin{array}{c}\text { Consumo uniforme } \\
(\text { días })\end{array}$ \\
\hline 2 & $12.6 \pm 1.0^{\mathrm{a}}$ \\
3 & $12.0 \pm 1.0^{\mathrm{a}}$ \\
4 & $10.0 \pm 1.0^{\mathrm{b}}$ \\
\hline${ }^{\mathrm{a}, \mathrm{b}}$ Superíndices diferentes indican diferencia \\
estadística $(\mathrm{p}<0.05)$
\end{tabular}

Cuadro 4. Consumo promedio de concentrado en los 15 días del estudio, según la edad de las crías de alpaca $(\mathrm{n}=10$ por grupo)

\begin{tabular}{cc}
\hline $\begin{array}{c}\text { Grupos } \\
\text { (meses de edad) }\end{array}$ & $\begin{array}{c}\text { Consumo } \\
(\mathrm{g})\end{array}$ \\
\hline 2 & $46.3 \pm 15.2^{\mathrm{ab}}$ \\
3 & $56.7 \pm 18.5^{\mathrm{a}}$ \\
4 & $34.4 \pm 9.7^{\mathrm{b}}$ \\
\hline $\mathrm{a}, \mathrm{b}$ Superíndices diferentes indican dife-rencia \\
estadística $(\mathrm{p}<0.05)$
\end{tabular}

gistrado en los animales de 3 meses de edad. Estas diferencias podrían deberse a una menor cautela (Provenza et al., 1992) en la ingestión del concentrado por los animales de tres meses de edad con respecto a los de cuatro meses. Si bien el menor tiempo de aceptación de alimento concentrado se registró en los animales de cuatro meses, se evidencia que perderían interés por un nuevo alimento, pudiendo interpretarse que, a esta edad, las alpacas mostrarían mayor renuencia hacia la ingestión de un nuevo alimento por el fenómeno de la neofobia, reduciéndose la ingesta del concentrado. Por otro lado, también podría deberse a que los animales de mayor edad perderían el interés por el alimento nuevo, mostrando renuencia a su ingestión, porque consumen una mayor cantidad de pasto, el cual sería más apetecible que el concentrado. Los animales de cuatro meses tienen más desarrollada la capacidad de utilización de los forrajes que los de menor edad y serían más eficientes en la utilización de las paredes celulares y bajo contenido nitrogenado de los pastos naturales (Prud'hon et al., 1993; López et al., 2000).

El presente estudio se diseñó de tal manera que los animales no pudieran tener una dieta exclusiva de concentrado, por lo que el consumo fue en absoluto voluntario, considerando que los animales estuvieron encerrados y expuestos al concentrado por tres horas al día, pero luego salían a pastar y 
a lactar. Los animales, de esa forma, tuvieron posibilidades de elección entre el alimento concentrado, el pasto y la leche materna, teniendo libertad de expresar sus patrones de elección. Por lo tanto, las variables medidas responden a una experiencia temprana. No obstante, se señala que estos hallazgos merecen estudios continuados a largo plazo, con el fin de determinar la persistencia de la memoria y el grado de aceptación del mismo alimento después de un periodo más prolongado.

\section{Conclusiones}

La edad apropiada de las alpacas para el aprendizaje temprano de consumo de concentrado es a los tres meses de edad, debiendo exponerse al consumo del concentrado por un periodo mayor de 11 días para que el alimento sea considerado parte de la dieta diaria.

\section{Agradecimientos}

Al Dr. Enrique Flores Mariazza, por su apoyo en la planificación del trabajo de investigación, y al personal administrativo y técnico de la UP Conocancha de la SAIS Pachacutec, por su apoyo en la fase de campo del estudio.

\section{Literatura Citada}

1. Burrit EA, Provenza FD. 1991. Ability of lambs to learn with a delay between food ingestion and consequences given meals containing novel and familiar foods. Appl Anim Behav Sci 32: 179-189. doi: 10.1016/S0168-1591(05)80041-3

2. Burrit EA, Provenza FD. 2000. Role of toxins in intake of varied diets by sheep. J Chem Ecol 26: 1991-2005. doi: 10.1023/A:1005565228064

3. Dam Van Tien, Le Thi Van Ha. 2000. Manipulation of feeding behaviour for ruminant production: techniques for better use of novel feeds for goat production in central part of Vietnam. Workshop-Seminar «Making better use of local feed resources» SAREC-UA. [Internet]. Available in: http://www. mekarn.org/sarpro/tien.htm

4. Dam Van Tien. 2003. Effects of experience in the pre-weaning period on food familiarity and intake in goats: transmission of preference for rice straw via mother's milk or by social facilitation. In: Proc Final National SeminarWorkshop on Sustainable Livestock Production on Local Feed Resources. HUAF-SAREC, Hue City. [Internet]. Available in: http://www.mekarn.org/ sarec03/Tien1.htm

5. Launchbaugh KL, Provenza FD. 1991. Learning and memory in grazing livestock application to diet selection. Rangelands 13: 242-244.

6. López, A, Raggi LA. 1992. Requerimientos nutritivos de camélidos sudamericanos: llamas (Lama glama) y alpacas (Lama pacos). Arch Med Vet 24: 121-130.

7. López VA, Morales S, Cabrera C, Urra CX. 2000. Ingestión y digestibilidad aparente de forrajes por la llama (Lama glama): I.- Heno de alfalfa (Medicago sativa) y paja de trigo (Triticum aestivum) en diferentes proporciones. Arch Med Vet 32: 201-208. doi: 10.4067/ S0301-732X2000000200007

8. Mirza SN, Provenza FD. 1994. Socially induced food avoidance in lambs: direct or indirect maternal influence? J Anim Sci 72: 899-902. doi: 10.2527/ 1994.724899x

9. Provenza FD, Balph DF. 1987. Diet learning by domestic ruminants: theory, evidence and practical implications. Appl Anim Behav Sci 18: 211-232. doi: 10.1016/0168-1591(87)90218-8

10. Provenza FD, Pfister JA, Cheney CD. 1992. Mechanisms of learning in diet selection with reference to phytotoxicosis in herbivores. J Range Manage 45: 36-45. 
11. Provenza FD. 1995. Tracking variable environments: there is more than one kind on memory. J Chem Ecol 21: 911923. doi: 10.1007/BF02033798

12. Provenza FD, Launchbaugh $K L$. 1999. Foraging on the edge of chaos. In: Grazing behavior of livestock and wildlife. USA: Univ of Idaho. 1-12 p.

13. Provenza FD. 2003a. Twenty-five years of paradox in plant-herbivore interactions and «sustainable» grazing management. Rangelands 25: 4-15.

14. Provenza FD. 2003b. Foraging behavior: managing to survive in a world of change. Behavioral principles for human, animal, vegetation, and ecosystem management. Utah State University. 62 p. [Internet]. Available in: http://works.bepress.com/ frederick_provenza/155/

15. Provenza FD, Villalba JJ. 2006. Foraging in domestic vertebrates: linking the internal and external milieu. In: Bels VL (ed). Feeding in domestic vertebrates: from structure to function. Oxfordshire, UK: CABI Publ. p 210-240.

16. Prud'hon M, Cordesse RS. De Rouville $S$, Thimonier J. 1993. Les camélidés sud-américains: le point des connaissances. INRA Prod Anim 6(1): 5-15.

17. San Martín F. 1991. Alimentación y nutrición. En: Fernández-Baca S (ed). Avances y perspectivas del conocimiento de los camélidos sudamericanos. Santiago, Chile: FAO. Oficina Regional para América Latina y el Caribe. p 213-261.

18. San Martin F. 1994. Avances y alternativas de alimentación para los camélidos sudamericanos. Investigaciones Pecuarias. Rev Invest Pec 7(2). Disponible en: http://sisbib.unmsm. edu.pe/bvrevistas/veterinaria/v07_n2/ avancesya.htm 